\title{
Usefulness of Quantitative Bone Single-Photon Emission Computed Tomography/Computed Tomography for Evaluating the Treatment Response of Bone Metastasis in a Lung Cancer Patient
}

\author{
Kazuhiro Kitajima ${ }^{a}$ Tatsuya Tsuchitani $^{\text {b }}$ Yoshiyuki Takahashib \\ Toshiyuki Minamic Takashi Yokoic Akifumi Nakamurad $^{\mathrm{c}}$ \\ Masaki Hashimoto ${ }^{d}$ Kozo Kuribayashic ${ }^{c}$ Takashi Kijima $^{c}$

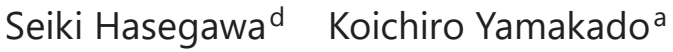 \\ a Department of Radiology, Hyogo College of Medicine, Hyogo, Japan; \\ ${ }^{b}$ Department of Radiological Technology, Hyogo College of Medicine College Hospital, \\ Hyogo, Japan; ' Division of Respiratory Medicine, Department of Internal Medicine, \\ Hyogo College of Medicine, Hyogo, Japan; ${ }^{\mathrm{d}}$ Department of Thoracic Surgery, Hyogo \\ College of Medicine, Hyogo, Japan
}

\section{Keywords}

Bone metastasis · Treatment response - Bone scintigraphy · Quantitative SPECT/CT .

Standardized uptake value

\begin{abstract}
We report a case of bone metastasis arising from lung cancer, including quantitative values obtained with bone single-photon emission computed tomography/computed tomography (SPECT/CT), which were useful to evaluate the treatment response. The first bone SPECT/CT during pembrolizumab therapy for lung cancer recurrence showed intense ${ }^{99 \mathrm{mT}} \mathrm{T}-\mathrm{HMDP}$ uptake of the right femur head and mild uptake of the left ribs. After the palliative radiotherapy for the right femur head metastasis and chemotherapy, the second bone SPECT/CT showed a decrease in focal uptake of the right femur hip and increasing uptake of the left ribs. There was also new uptake appearance in the sternum, right rib, spine (Th2, Th9, Th12, L4, S1), and bilateral pelvic bone (left ilium, acetabular cartridge, femur, right ilium and ischium). The change of maximum standardized uptake values (SUVmax) for the right femur head and left third and eighth rib bony metastases were $-72.6 \%$ (from 22.96 to 6.28 ), $+407.7 \%$ (from 2.97 to 15.08 ), and $+229.2 \%$ (from 2.60 to 8.56 ), respectively. The change of whole-lesion metabolic bone volume and total bone uptake was $+235.4 \%$ (from 22.75 to $76.3 \mathrm{~cm}^{3}$ ) and $+219.1 \%$ (from 205.0 to 654.09 ), respectively. Two quantitative bone SPECT/CT images clearly showed the good response of femur head metastasis due to radiotherapy, and progression of other bone metastases regardless of chemotherapy.




\section{Introduction}

Radionuclide bone scintigraphy with technetium $99 \mathrm{~m}\left({ }^{99 \mathrm{~m}} \mathrm{Tc}\right)$ can evaluate osteoblastic activity and is a widely used valuable technique for the assessment of disease extent and treatment response in patients with bone metastasis. However, abnormal uptake in bone observed with planar bone scintigraphy cannot be easily and reliably quantified, and semiquantitative visual methods are not adequately sensitive to measure the actual response, even with images acquired using single-photon emission computed tomography (SPECT). On the other hand, recent advances have resulted in the integration of computed tomography (CT) for attenuation correction together with a sophisticated reconstruction technique, which has enabled quantitative measurements with SPECT/CT suitable for derivation of the standardized uptake value (SUV) [1-3]. Several groups have demonstrated the clinical application of quantitative bone SPECT/CT parameters (SUV and uptake volume) in the management of malignant bony disease [4-8] as well as positron emission tomography (PET). It is considered that quantitative SPECT/CT may soon have an enormous effect as an imaging biomarker in the field of modern nuclear medicine and for clinical practice. Here, we report a case of lung cancer in which quantitative values were determined with bone SPECT/CT measurements and found to be useful to evaluate the treatment response of bone metastasis.

\section{Case Report}

A 64-year-old female underwent radical right lower lobectomy surgery due to lung cancer (squamous cell carcinoma, pT1aN0M0) 6 years ago. However, 1 year ago, mediastinal nodal metastasis was detected by ${ }^{18} \mathrm{~F}$-fluorodeoxyglucose $\left({ }^{18} \mathrm{~F}-\mathrm{FDG}\right) \mathrm{PET} / \mathrm{CT}$ and proved pathologically to have mediastinal nodal metastasis by mediastinoscopy (the expression of programmed cell death 1 was 95\%). Pembrolizumab as the first-line therapy continued for 1 year (15 cycles); however, the patient complained of right hip pain. Bone SPECT/CT with ${ }^{99 \mathrm{~m}} \mathrm{Tc}$ hydroxymethylene diphosphonate ${ }^{99 \mathrm{~m}} \mathrm{Tc}-\mathrm{HMDP}$, Nihon Medi-Physics Co. Ltd, Tokyo, Japan) showed focal and intense uptake by the right femur head and faint uptake by the left ribs (third and eighth; Fig. 1a), and bony metastasis was confirmed by MRI. She received palliative radiotherapy (20 Gy/5 Fr) for the right femur head metastasis. Carboplatin and nab-paclitaxel was started as the second-line therapy; however, 4 months later CT showed the appearance of lung metastasis and adrenal metastasis. Fluorouracil was started as the third-line therapy; however, 2 months later a second bone SPECT/CT (8 months after the first bone SPECT/CT) showed a decrease of the right femur head uptake, increase in the uptake by the left ribs, and the appearance of new uptake in the sternum, right rib, spine (Th2, Th9, Th12, L4, S1), and bilateral pelvic bone (left ilium, acetabular cartridge, femur, right ilium and ischium; Fig. 1b). The serum CEA and CA19-9 on the first and second ${ }^{99 \mathrm{~m}} \mathrm{Tc}-\mathrm{HMDP}$ bone SPECT/CT increased from 41.9 to $602 \mathrm{ng} / \mathrm{mL}(1,336.8 \%$ increase) and 267 to $8,555 \mathrm{U} / \mathrm{mL}$ $(3,104.1 \%$ increase $)$, respectively.

Two bone SPECT/CT scans using an integrated SPECT/CT system (Discovery NM/CT 670, GE Healthcare) equipped with a low-energy high-resolution collimator were performed $3 \mathrm{~h}$ after intravenous injection of $555 \mathrm{MBq}$ of ${ }^{99 \mathrm{~m}} \mathrm{Tc}-\mathrm{HMDP}$. SPECT/CT data were analyzed using a commercially available software package (GI-BONE, Aze Co. Ltd, Tokyo, Japan), which provides statistics for various SUVs, such as the maximum, peak, and mean SUV, as well as the metabolic bone volume (MBV) and total bone uptake (TBU) [6]. The SUVmax was defined as the maximum concentration in the target lesion: (maximum radioactivity/voxel volume)/(injected radioactivity/body weight). The SUVpeak was defined as the average activity concentration within a $1-\mathrm{cm}^{3}$ spherical volume of interest (VOI) centered on the "hottest focus" within the tumor. The

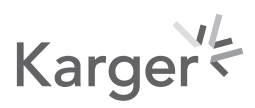




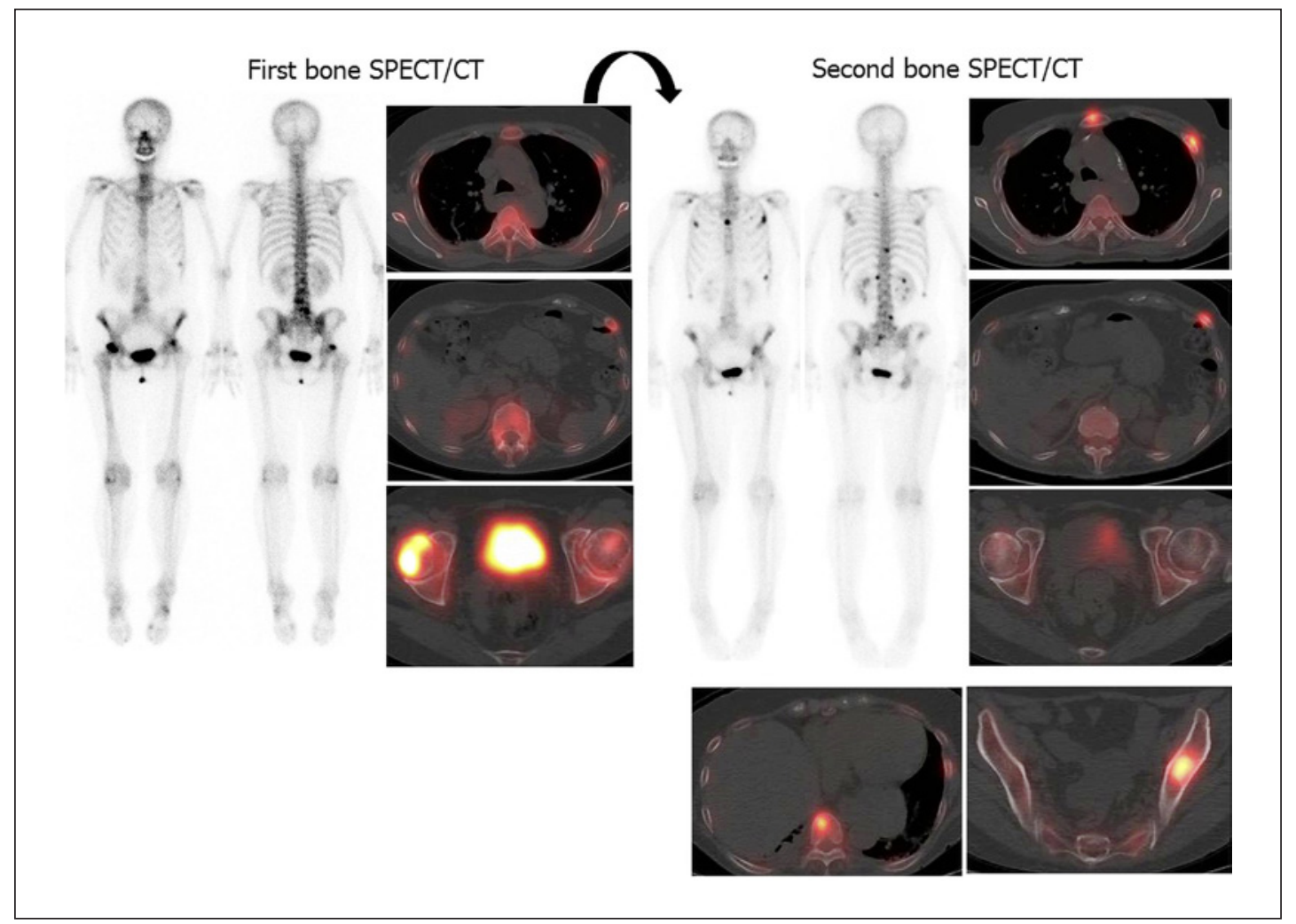

Fig. 1. Two bone SPECT/CT scans of a 64-year-old female with bone metastases from lung cancer. The first bone SPECT/CT image shows strong focal ${ }^{99 \mathrm{~m}} \mathrm{Tc}$-hydroxymethylene diphosphonate ( ${ }^{99 \mathrm{~m}} \mathrm{Tc}$-HMDP) uptake in the right femur head and mild uptake by the left ribs. The post-treatment (palliative radiotherapy for the right femur head metastasis and chemotherapy) bone SPECT/CT shows a decrease in focal ${ }^{99 \mathrm{~m}} \mathrm{Tc}$-HMDP uptake in the right femur head and an increase of uptake by the left ribs, and the appearance of new uptake in the sternum, right rib, spine (Th2, Th9, Th12, L4, S1), and bilateral pelvic bone (left ilium, acetabular cartridge, femur, right ilium and ischium).

SUVmean was defined as: (total radioactivity/VOI volume)/(injected radioactivity/body weight). The MBV $\left(\mathrm{cm}^{3}\right)$ was the calculated volume obtained by the radiopharmaceutical accumulation by placing the tumor VOI with the margin threshold set at $40 \%$ of the SUVmax. The TBU was then calculated as the SUVmean $\times$ MBV. The bone scan index (BSI) was automatically calculated by two-dimensional planar imaging (anterior and posterior images) [9].

The change of SUVmax, SUVpeak, SUVmean, MBV, and TBU for right femur head metastasis was $-72.6 \%$ (from 22.96 to 6.28 ), $-70.4 \%$ (from 19.66 to 5.82 ), $-64.5 \%$ (from 12.98 to 4.61), $-35.1 \%$ (from 14.70 to $9.54 \mathrm{~cm}^{3}$ ), and $-77.0 \%$ (from 190.81 to 43.97 ), respectively (Fig. 2). The change of SUVmax, SUVpeak, SUVmean, MBV, and TBU for left third rib metastasis was $+407.7 \%$ (from 2.97 to 15.08 ), $+416.7 \%$ (from 2.33 to 12.04 ), $+392.8 \%$ (from 1.80 to 8.87 ), $+31.0 \%$ (from 5.46 to $7.15 \mathrm{~cm}^{3}$ ), and $+545.1 \%$ (from 9.82 to 63.35 ), respectively. The change of SUVmax, SUVpeak, SUVmean, MBV, and TBU for left eighth rib metastasis was $+229.2 \%$ (from 2.60 to 8.56 ), $+236.7 \%$ (from 2.10 to 7.07 ), $+226.2 \%$ (from 1.68 to 5.48 ), $+30.9 \%$ (from 2.59 to $3.39 \mathrm{~cm}^{3}$ ), and $+325.2 \%$ (from 4.37 to 18.58 ), respectively. The SUVmax, SUVpeak, SUVmean, MBV, and TBU of left ileum metastasis on the second bone SPECT/CT were 19.91, 18.56, 12.27, $21.53 \mathrm{~cm}^{3}$, and 264.12, respectively. The SUVmax, SUVpeak, SUVmean, MBV, and TBU of sternum metastasis on the second bone SPECT/CT were 12.77, $10.14,7.49,5.09 \mathrm{~cm}^{3}$, and 38.11, respectively. The SUVmax, SUVpeak, SUVmean, MBV, and 


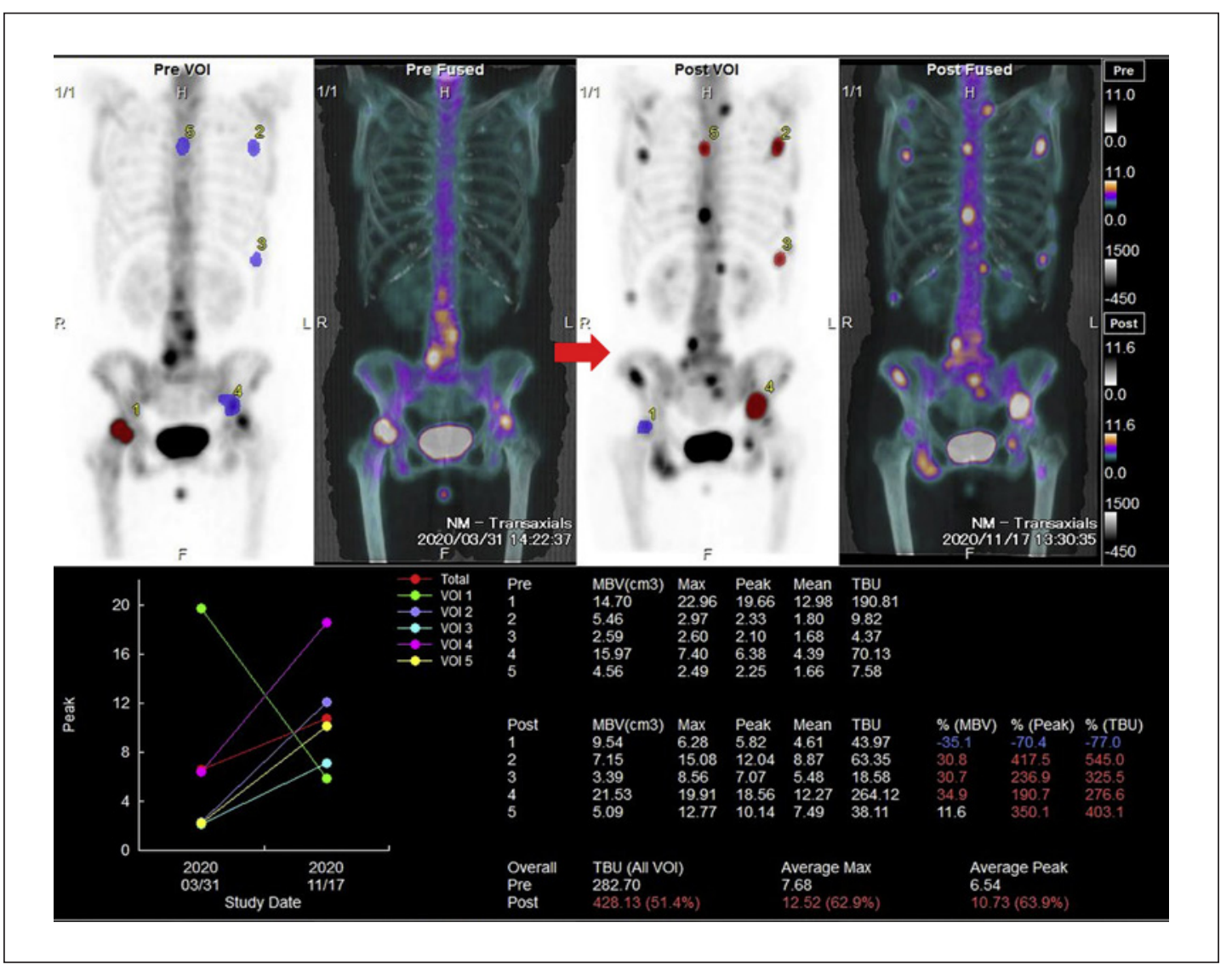

Fig. 2. Three-dimensional quantitative values of bone SPECT/CT. The change of SUVmax for the right femur head and left third and eighth rib bony metastases was $-72.6 \%$ (from 22.96 to 6.28 ), $+407.7 \%$ (from 2.97 to 15.08), and $+229.2 \%$ (from 2.60 to 8.56 ), respectively. The SUVmax values of the left ileum, right ileum, sacrum, sternum, and thoracic vertebrae Th9 metastasis on the second bone SPECT/CT were 19.91, 12.90, 9.11, 12.77, and 17.25, respectively. The change of whole-lesion MBV and TBU was $+235.4 \%$ (from 22.75 to 76.3 $\mathrm{cm}^{3}$ ) and $+219.1 \%$ (from 205.0 to 654.09 ), respectively.

TBU of right ileum metastasis on the second bone SPECT/CT were 12.90, 11.30, 7.88, 10.51 $\mathrm{cm}^{3}$, and 82.8, respectively. The SUVmax, SUVpeak, SUVmean, MBV, and TBU of sacrum metastasis on the second bone SPECT/CT were 9.11, 8.47, 5.79, $11.95 \mathrm{~cm}^{3}$, and 69.21 , respectively. The SUVmax, SUVpeak, SUVmean, MBV, and TBU of thoracic vertebrae Th9 metastasis on the second bone SPECT/CT were 17.25, 14.43, 10.36, $7.14 \mathrm{~cm}^{3}$, and 73.95, respectively. The change of whole-lesion MBV and TBU was $+235.4 \%$ (from 22.75 to $76.3 \mathrm{~cm}^{3}$ ) and $+219.1 \%$ (from 205.0 to 654.09 ), respectively. On the other hand, the change of BSI was $+797.5 \%$ (from 0.4 to $3.59 \%$ ). Two quantitative bone SPECT/CT images clearly showed the good response of the femur head metastasis to radiotherapy, and progression of other bone metastases regardless of chemotherapy.

\section{Discussion}

Traditionally, gamma camera imaging has been used in nuclear medicine for two-dimensional planar imaging, with bone scintigraphy with ${ }^{99 \mathrm{~m}} \mathrm{Tc}$ phosphonates a typical example of such a technique. Using results of robust algorithms of CT-based three-dimensional attenuation 
correction, scatter correction, and resolution recovery, SPECT/CT generates imaging voxels denoted as units of radioactivity per volume (i.e., $\mathrm{kBq} / \mathrm{mL}$ ). This is fundamentally different from traditional nuclear imaging methods, such as planar scintigraphy, SPECT, and nonquantitative SPECT/CT, which use counts per second for their imaging units. With quantitative SPECT/ $\mathrm{CT}$, lesion radioactivity can be normalized for injected radioactivity, resulting in quantitative parameter values, such as percent injected dose and SUV [1-3], Quantitative bone SPECT/CT with SUV [4-8], MBV [5, 6], and TBU [5, 6] was subsequently developed following the successful clinical application of SPECT/CT findings in patients with malignant skeletal disease.

Beck et al. [4] investigated changes in SUV before and after treatment in 16 breast cancer and 3 prostate cancer patients (hormonal therapy and bisphosphonates) using quantitative skeletal SPECT/CT with ${ }^{99 m}$ Tc-diphosphono-propanedicarboxylic acid (99m Tc-DPD). They reported mean SUVpeak and SUVmean values for 52 bone metastasis sites prior to treatment of $20.4 \pm 20.8$ and $5.6 \pm 1.9$, which were decreased following treatment to $16.4 \pm 11.5(19.6 \%$ reduction) and $4.9 \pm 2.2$ (12.5\% reduction), respectively. Umeda et al. [5] evaluated the usefulness of three-dimensional TBU for evaluating the active bone metastatic burden in 9 prostate cancer patients who underwent Ra-223 therapy and found that the change in TBU derived from the established threshold value (SUV > 7.0) was more accurate and sensitive than the change in classic BSI in those cases. Furthermore, Kitajima et al. [6] reported a case of periosteal osteosarcoma in which SUVmax, SUVpeak, SUVmean, MBV, and TBU values shown by quantitative bone SPECT/CT with ${ }^{99 \mathrm{~m}} \mathrm{Tc}$-HMDP were useful to evaluate the treatment response to preoperative chemotherapy. Kuji et al. [7] evaluated SUVmax, SUVpeak, SUVmean of normal vertebral body, degenerative change, and bone metastases in 170 patients with prostate cancer undergoing bone SPECT/CT with ${ }^{99 \mathrm{~m}} \mathrm{Tc}$-methylene diphosphonate ( $\left.{ }^{99 \mathrm{~m}} \mathrm{Tc}-\mathrm{MDP}\right)$ and demonstrated that SUVmax was $7.58 \pm 2.42$ for thoracic normal vertebral body, $8.12 \pm 12.24$ for lumbar normal vertebral body, $16.73 \pm 6.74$ for degenerative changes, and $40.90 \pm 33.46$ for bone metastases. The SUVpeak values of these four corresponding figures were $6.51 \pm 2.12$, $6.68 \pm 1.93,13.74 \pm 4.52$, and $34.24 \pm 29.91$, respectively. The SUVmean values of these four corresponding figures were $4.62 \pm 1.68,4.54 \pm 1.38,9.47 \pm 3.94$, and $24.59 \pm 21.19$, respectively. The SUVs of the bone metastasis group were significantly $(p<0.001)$ greater than of the other three groups. In receiver-operator characteristic (ROC) analyses for bone metastases by SUVs (SUVmax, SUVpeak, and SUVmean) demonstrating the diagnostic accuracy of SUVs for discriminating bone metastasis from degenerative changes in hot foci, the area under ROC curves values were $0.840,0.817$, and 0.845 in the patient-based mode. Tabotta et al. [8] analyzed 264 bone metastases from 26 prostate cancer patients and 24 spinal and pelvic osteoarthritic lesions from 13 patients without any cancer, using quantitative skeletal SPECT / CT with ${ }^{99 \mathrm{~m}}$ Tc-DPD, and demonstrated a mean SUVmax and SUVmean of $34.6 \pm 24.6$ and 20.8 \pm 14.7 for bone metastases versus $14.2 \pm 3.8$ and $8.9 \pm 2.2$ for spinal and pelvic osteoarthritic lesions, respectively. The SUVmax and SUVmean were both significantly different between the bone metastases and osteoarthritic groups ( $p<0.0001)$. Using a SUVmax cutoff of 19.5 for prostate cancer bone metastases in the spine and pelvis, the sensitivity, specificity, and positive and negative predictive values were $87,92,99$, and $49 \%$, respectively.

\section{Conclusion}

We have found that three-dimensional quantitative bone SPECT/CT with SUVmax, SUVpeak, SUVmean, MBV, and TBU is a noninvasive useful modality to evaluate the activity of bone metastasis as well as the treatment response. Additional studies with greater numbers of patients are needed to define its impact for clinical evaluation of the treatment response of bony lesions.

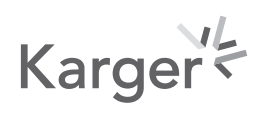




\section{Case Reports in Oncology}

\begin{tabular}{l|l}
\hline Case Rep Oncol 2021;14:391-396 \\
\hline DOI: 10.1159/000513907 & $\begin{array}{l}\text { ○ 2021 The Author(s). Published by S. Karger AG, Basel } \\
\text { www.karger.com/cro }\end{array}$ \\
\hline
\end{tabular}

Kitajima et al.: Treatment Response of Bone Metastasis by Quantitative Bone SPECT/CT

\section{Statement of Ethics}

This report complies with the guidelines for human studies and includes evidence that the research was conducted ethically in accordance with the World Medical Association Declaration of Helsinki. The authors have no ethical conflicts to disclose. Written informed consent was obtained from the patient for publication of this case report and any accompanying images.

\section{Conflict of Interest Statement}

The authors have no conflicts of interest to declare.

\section{Funding Sources}

This work was supported by JSPS KAKENHI grant number 19 K08187.

\section{Author Contributions}

Concept and design, and drafting of the manuscript: Ka.K. Acquisition of data: T.T., Y.T., T.M., T.Y., Ko.K., T.K., A.N., M.H., S.H. Critical revision of the manuscript for important intellectual content: Ka.K., K.Y. All authors approved final version of the manuscript.

\section{References}

1 Zeintl J, Vija AH, Yahil A, Hornegger J, Kuwert T. Quantitative accuracy of clinical 99mTc SPECT/CT using ordered-subset expectation maximization with 3-dimensional resolution recovery, attenuation, and scatter correction. J Nucl Med. 2010;51(6):921-8.

2 Bailey DL, Willowson KP. An evidence-based review of quantitative SPECT imaging and potential clinical applications. J Nucl Med. 2013;54(1):83-9.

3 Gnesin S, Leite Ferreira P, Malterre J, Laub P, Prior JO, Verdun FR. Phantom validation of Tc-99m absolute quantification in a SPECT/CT commercial device. Comput Math Methods Med. 2016;2016:4360371.

4 Beck M, Sanders JC, Ritt P, Reinfelder J, Kuwert T. Longitudinal analysis of bone metabolism using SPECT/CT and 99mTc-diphosphono-propanedicarboxylic acid: comparison of visual and quantitative analysis. EJNMMI Res. 2016;6(1):60.

5 Umeda T, Koizumi M, Fukai S, Miyaji N, Motegi K, Nakazawa S, et al. Evaluation of bone metastatic burden by bone SPECT/CT in metastatic prostate cancer patients: defining threshold value for total bone uptake and assessment in radium-223 treated patients. Ann Nucl Med. 2018;32(2):105-13.

6 Kitajima K, Futani H, Fujiwara M, Minakawa G, Osugi Y, Tsuchitani T, et al. Usefulness of quantitative bone single photon emission computed tomography/computed tomography for evaluating response to neoadjuvant chemotherapy in a patient with periosteal osteosarcoma. Cureus. 2018;10(11):e3655.

7 Kuji I, Yamane T, Seto A, Yasumizu Y, Shirotake S, Oyama M. Skeletal standardized uptake values obtained by quantitative SPECT/CT as an osteoblastic biomarker for the discrimination of active bone metastasis in prostate cancer. Eur J Hybrid Imaging. 2017;1(1):2.

8 Tabotta F, Jreige M, Schaefer N, Becce F, Prior JO, Nicod Lalonde M. Quantitative bone SPECT/CT: high specificity for identification of prostate cancer bone metastases. BMC Musculoskelet Disord. 2019;20(1):619.

9 Shimizu A, Wakabayashi H, Kanamori T, Saito A, Nishikawa K, Daisaki H, et al. Automated measurement of bone scan index from a whole-body bone scintigram. Int J Comput Assist Radiol Surg. 2020;15(3):389-400. 